It is to be regretted that at the moment of the examination of the ten-year-old child we did not examine the dark adaptation. The parents refused afterwards to re-visit the clinic for this purpose.

In discussing this case and especially the fundus alterations suggesting scars of chorioretinitis I might draw attention to similar cases described elsewhere, particularly to the case of Magnus (Graefe's Archiv., 118, 1920) who studied a case of bilateral pseudo-glioma caused by fibrous tissue behind the lens with arteria hyaloidea persistens and microphthalmos. The second eye could be partly examined ophthalmoscopically and the aspect is described as follows : discoloured and depigmentated areas are visible that remind one of choroiditis and are localised in the layer of the pigment epithelium. Nowhere retinal vessels were visible, although no detachment existed in this part !

This case shows that the fundus appearance found in our case does not necessarily prove an inflammatory process.

FURTHER LITERATURE

HEINE.-Arch. f. Ophthal., Bd. LVIII, 1904.

HEINE.-Zeitschr. f. Augenheilk., Bd. LI, 1923.

\title{
SOME CLINICAL NOTES ON THE NATURE OF THE RETINAL VENOUS PULSE
}

\author{
BY \\ N. PINES \\ LONDON
}

\section{Description}

THE same method was used as in the investigation of retinal vascular disease-a 12 volt red-free light worked with a current of 20 volts or more. This is essential as the brilliancy of the over-heated wire increases the red-free part of the spectrum and the more overcharged the lamp the better it is for the resulting illumination. The patient would generally experience great discomfort if this intense light were to fall unguarded on to the retina, but if the red-free filter is placed in such a position that all the light emanating from the bulb is coloured, it will mitigate this inconvenience.

Commencing from the periphery, in the primary branches of the veins and, in favourable cases, in even the secondary branches, especially the thicker ones, the venous pulse was discovered in all patients except where an obstruction in the optic nerve or further beyond it produced an engorgement of the veins. It is much 
more noticeable in children and in the young ceteris paribus, but is not much affected by age or disease. It becomes more pronounced nearer the disc and on the disc itself it presents two varieties. One is the same as on the periphery and may be called the ordinary basic pulse. The second is the well-known type of piston-like movement that we will call the spontaneous venous pulse. Both are of locomotor character, i.e., no displacement of the venous trunk is caused by them. On the periphery one can discern the venous pulse by carefully watching the vein, or by noticing the light reflexes already described, as produced by the column of blood inside the vessel. If a comparison be permissible it resembles the respiratory movement of the abdomen of a small lizard when motionless. In all patients the number of widenings and narrowings of a vessel on the periphery (both counted as one) corresponds in number to the pulsations of the temporal artery, but they are slightly slower. This regularity is still preserved on the disc in the majority of cases, but in some patients a kind of back-flow of blood suddenly appears and the movements of the venous walls are not as smooth as before. If one can trace the venous pulse deeply on the disc in many cases one can see both veins pulsating in a kind of spontaneous venous pulse, but not in such a clear piston-like manner as the original second type.

If a vein, even a sharply pulsating one, is crossed on the disc by an artery, the latter may be a very thin one, the venous pulse is usually but not always, stopped or at least considerably diminished, at the point of crossing. A sister vein, but not a crossed one, will show a spontaneous pulsation for a longer distance because it is not impeded by the crossing artery. It may be argued and quite correctly, that this sister vein may simply dip in the central vein at a sharper angle, but this would hardly apply in all cases. The basic venous pulses, on the contrary, are seldom impeded or influenced by an arterial crossing, even in a highly sclerotic eye, unless the vein is definitely crushed, has white stripes at the point of crossing and subsequent banking and deflection. If a vein crosses an artery, even if the vein is flattened and the blood stream becomes so thin that the artery can be seen through the super-imposed vessel, the spontaneous venous pulse, if present, is more distinct and brisk. This, however, only occurs when the vein bends over the artery and then sharply dips into the disc, the artery serving only as an additional factor to impress the sharpness of the bend on the vein. If, on the other hand, the vein crosses the artery somewhere near the periphery of the disc and then dips sharply into the disc, the spontaneous pulse will only appear at the latter point, the former being powerless to produce it as the vein is not bent sharply, but 
if the vein is bent sharply over the artery then the pulse is distinctly of the spontaneous type especially if the angle of the bend is very sharp. At this point it is important to note that the observer sees the crossing from the obtuse side of the angle but striking at the top of the apex in the sagittal direction. It may also be observed when the vein bends sharply over the edge of the disc, but I did not see a spontaneous venous pulse in cases with glaucomatous excavations. One must bear in mind that all the above remarks are only more or less accurate, as the combination of veins and arteries on the disc and their designs are protean and numerous.

The second type.-The piston-like movement of the spontaneous venous pulse is a beautiful phenomenon; if once detected it is constantly present in the same vessels, the number of pulsations being identical with the beat of the temporal artery, but slightly posterior in time. It is regular and does not noticeably change in character as sometimes happens in the first variety. Briskly spontaneous on the disc, especially before the artery dips into the disc, it is of the first type on the periphery. It is especially noticeable if the artery disappears into the disc at an angle of $90^{\circ}$ or less. The sharper the angle formed with the disc the better. Although the character of the spontaneous pulse is constant for a definite vein its intensity may be different for veins of the same size and direction in different subjects. One or more veins in the same eye may show the spontaneous pulse and the rest the ordinary one. Otherwise, all veins show the spontaneous pulse, or else the ordinary one. It is interesting sometimes to watch a few major trunks flow majestically and contract with dignity on the disc, whilst a small one, usually independent, is very busy with spontaneous pulsation. Each eye in the same patient has its own combination. By applying digital pressure or using Bailliart's ingenious dynamometer, it is well-known that one can produce some changes in the pictures. Some or all the veins will change their ordinary venous pulse into a spontaneous one, the originally spontaneous pulsating vein only intensifying its action and blanching at the end of every pulsation, but never changing from the second to the first type. A few or all the veins of the first type will collapse altogether, but will never change to the second one, and the spontaneous vein will collapse also, but will not change to the first type. For the purpose of this paper it was more important to note the percentage of eyes with a spontaneous venous pulse and then to distinguish between those where only a few or all the veins were thus acting. This, together with the difference in technique, is the probable explanation of the fact that my figures may not be identical with those of such authorities, to mention only two from among a very large number, as R. H. Elliot and Bailliart. 
According to Elliot, the spontaneous venous pulse was seen in both eyes in 46 per cent., absent in 40 per cent. and present in one eye only in 14 per cent. Of the group of 46 per cent., the pulse was even in both eyes in 28 per cent. and uneven in 18 per cent. Of the 40 per cent. where the spontaneous pulse was absent, in 12 per cent. it could be elicited in both eyes by pressing and in 18 per cent. in one eye only. In 200 eyes the spontaneous pulse was present in 49 per cent. and absent in 51 per cent. To quote Elliot's words, "one can never detect the venous pulse beyond the papillary limit." This may be accounted for in three ways. (a) The vessels and their movements show up, on the optic disc. (b) On the retina they are imbedded in its structure, whilst on the disc they lie comparatively free (Bailliart). (c) The pulse has probably spent itself within the limits of the disc. It is usually accepted that every feature of these changes is transmitted from the auricles right along the columns of venous blood behind them.

Bailliart thinks that the spontaneous venous pulse exists when the venous pressure is slightly above or even equal to the intra-ocular tension. When the latter is much above the former the venous pulse can be produced by pressure on the globe. If the latter is inferior to the former massage of the globe will produce a venous pulse. The venous pulse is produced by the systolic wave of blood and aspiration by the diastole and systole of the auricle. Immediately after death both varieties of the venous pulse disappear. They usually cease quite suddenly and digital pressure only empties the veins. Once when I performed a venesection and whilst the patient was lying on the couch, immediately after death, as elicited by cessation of breathing, usual pupillary signs and abrupt cessation of the flow of blood from the cut vein, I saw for some moments very irregular beatings of the vein deep in the substance of the disc, similar to the flickering flame of a candle before it dies out.

Pressure in the retinal vessels.-Unlike the arterial and arteriocapillary system where the pressure is maintained by the-sit venia verbo-brute force of the systolic wave and consequent constriction of the distended peripheral vessels, the venous pressure is more elaborately organised. It depends first of all upon the transmission of the impetus-vis a tergo-from the arterial capillaries to the venous ones. The venous walls, although able to contract, and even to be controlled by a special centre in the brain, are not an essential item in the maintenance of the venous pressure and various factors are of importance in different parts of the body, such as, the respiratory movements of the chest, contractions of voluntary and involuntary muscles, 
diastole of the heart, etc. But if the science of the venous circulation is the Cinderella of modern clinical medicine it is a very precious item in the eyes of nature and no efforts are spared in maintaining it constantly and in any case, not below a certain level. Leaving aside the venous sinuses of the auricle of the heart, we find that in the eye, kidney, liver, spleen and perhaps the brain, a more or less elastic, but sometimes hardly distensible membrane is provided outside the organ to keep its structure maintained and at the same time to preserve the venous circulation.

Gauss saw the venous pulse in 91 cases out of 150 . It was not seen in 10 cases and could not be detected in the rest. Kurzes Handbuch der Ophthalmologie quotes only the authority of Bailliart and his figures. No spontaneous pulsation of the veins in 42 per cent., an intermittent one in 21 per cent. and a spontaneous venous pulse in 37 per cent.

\section{Statistics}

In Eyster's scheme the arterial pressure in man is $120 \mathrm{~mm}$., in arterioles $80 \mathrm{~mm}$., in capillaries $20 \mathrm{~mm}$., in the venules $10-15 \mathrm{~mm}$. and in the veins from plus $8 \mathrm{~mm}$. to minus $12 \mathrm{~mm}$. In BintonOpitz's experiments we find the same principle. The pressure in the saphenous vein is higher than that in the femoral; in the facial more than in the jugular. The figures for the abdominal cavity require special consideration. The highest pressure is in the mesenteric veins, probably because it is helped by the continuous peristaltic movements of the intestines. The same applies to the vena gastro-lienalis and lienalis. It cannot, however, be applied to the renal vein. It is probably the capsule which keeps the vascular pressure within the kidneys at a certain level, analogous to the sclera of the eye and the cranium to the head. It is therefore very tempting and justifiable to conclude that in maintaining the intravenous pressure nature has two ways-the helping action of the contracting muscles for the great majority of the veins-peripheral and central-and a more or less elastic capsule for the retina, kidney and brain, where the muscles are absent. On antomical grounds Sanderman comes to the same conclusion as regards the eyes. He is struck, moreover, on physiological grounds by the tense similarity with the kidney, as was the writer of this paper on pathological grounds. "In general there exists a marked similarity between the formation of urine by the glomeruli of the kidney and the formation of the aqueous by the ciliary body."

Again the close resemblance between the vascular physiology of the eye and of the kidney is very striking. Already in 
embryonic life according to Sanderman the arteries enter the bulb at the weakest part of sclera, namely around the disc, but the veins leave it at the strongest parts of the sclera. The result in embryo and adult will be identical. The fibres of the scleral coat gradually and increasingly compress the veins from within outwards so that the blood is allowed to escape only at a definite pressure. In this way nature not only forms and maintains the intra-ocular pressure, but from it results the continuous increase of the intravenous pressure, so that we cannot gauge the initial pressure in them were they to flow freely without an intra-ocular tension. Sanderman repeatedly found the pressure of the vortex veins to be $50-60 \mathrm{~mm}$. It will be shown later that this figure, if correct, is an enormous one for a vein.

It is obvious that the pressure in the central retinal vein must be higher than the intra-ocular tension, otherwise the outflow of the blood would be impeded. As soon as there is an obstruction somewhere on the disc or from there at any point to the heart, the retinal veins are engorged. The obstruction may be purely local or general, as in heart failure, or respiratory difficulties. In the latter case, the measurement of the brachial venous pressure by Villars' method will invariably show an increase of the venous pressure, but for the retina the basic rule is the same. Whatever the venous pressure it must be higher than the intra-ocular one, at least at the moment of the outflow of the blood through the lamina cribrosa. The difference is very small, it cannot even be measured by Bailliart's dynamometer. May I be allowed to say here apropos of this clever and ingenious, but not easily mastered method of the French master, that the present objections to its use are very similar to those raised against sphygmomanometry years ago, and are bound to follow in the same way, the sooner the better, both for the medical profession and the patients. Therefore for practical purposes we may take the venous retinal pressure as being identical with intra-ocular tension. Again it is obvious that the intracranial pressure must not be higher than the intra-ocular one, otherwise the retinal veins would be engorged, but in my opinion an attempt to measure the former through the venous pressure of the retina would not at present be very reliable as so many modifying factors interfere.

By the special method of passage of the vortex veins through the sclera, the eye is secure against sudden or free outflow of the venous blood and, therefore the venous pressure is constant. If in a normal person the systolic blood pressure in the brachial artery is, say, $120 \mathrm{~mm}$. of mercury and diastolic 80 , then in the arterioles it would be approximately two-thirds, i.e., 80/50. In the capillaries the systolic pressure is still further reduced, say, 
to $30 \mathrm{~mm}$., but the diastolic does not fall so quickly. From the physiological point of view the central retinal artery is an arteriole. By the ingenious and clinically correct method of Bailliart we can measure both on the living eye. I have not had any experience with the systolic pressure. It is of little importance clinically and the pressure applied on the eyes to produce it is very discomforting, but I often measure the diastolic and quite agree with Bailliart and his followers that in the ophthalmic artery on the disc it is normally approximately 0.45 of the brachial diastolic. What is it in the retinal capillaries? Slightly above the intra-ocular tension, otherwise the capillaries would be permanently closed. What would be the intra-vascular pressure in the venous capillaries? For the same reason slightly above the intra-ocular tension, i.e., identical with that of the capillary. But here we meet with a difficulty. We are certain that the arterial blood enters the eye without any hindrance as a certain pressure has to be applied to reach the level of the mean pressure inside the central artery. This in turn is evidence of the same free circulation in the capillaries, as any obstruction in their bed would immediately be compensated by a rise of the arteriolar pressure. But in the venous circulation we are not on such certain ground. It is feasible that the venous pressure may be below the intra-ocular tension. Then, when sufficient blood has been collected behind the obstruction the raised pressure causes an outflow of the blood and the vein partially collapses or narrows. What probably really happens is that the arterial capillaries open more widely, or in an augmented number constrict more vigorously, and supply more pressure, or transmit more arterial pressure towards the beginning of the venous capillaries, i.e., an increase of the vis a tergo. If one applies sufficient pressure on a healthy person's eye to collapse the veins on the disc the following will be seen. The pulsation of the artery becomes more pronounced and is noticeable further back on the retina than previously, but there is no blanching of the disc. The veins on the retina become slightly engorged, especially the primary branches and pulsate more vigorously. If a primary arterial branch crosses a similar venous one on the retina and not far from the disc, the proximal end of the vein may become narrowed and then the dorsal one (away from the crossing) is thicker and slightly enlarged, exactly as happens in the early stage of arterial venous compression, i.e., the artery crushes the vein, under supervision. When the pressure is removed the condition becomes normal.

Moreover, in some patients one can even see faint white shapes appearing on both sides of the spot where an artery crosses veins. In other patients they are absent. In my opinion, this is the result of a special perivascular membrane binding both vessels 
together, similar to a tunnel, and the different turgency or tightness of the membrane would explain the appearance of the white stripes in some patients. A similar condition occurs in favourable cases if a vein crosses an artery, but in a less pronounced degree, probably because of the lack of added weight of the superimposed artery. In both varieties, sometimes, the small white stripe may become visible and mildly bank and crush the vein, even if the artery branch cannot be seen. It means therefore that the pressure in the venous capillaries may be (1) primarily superior to the intra-ocular pressure, (2) primarily inferior, but increasing by the vis a tergo. Similar considerations would apply to the secondary and primary branches of the central vein. If, however, the vis a tergo is unavoidably the primary force the intensification of the venous pulse from the periphery of the retina towards the disc and in the same direction on the disc itself is, in my opinion, proof that here the outflow of venous blood is only possible by a gradual increase of internal vascular pressure, i.e., that the latter is slightly below the intra-ocular tension, but not sufficiently so for the latter to overcome the resistence of the venous wall and of the blood column itself and so to collapse the vein. Therefore, we have to deal with a vicious circle where the slightest outward interference will disturb the delicate vascular balance and immediately cause it to rearrange itself under new conditions and, therefore, all experimental measurements of the intravenous pressure on the animal will suffer from this primordial defect. This is why the clinical significance of both varieties of the venous pulse is nil at the present stage of our knowledge. It is rather a pity as the greater part of retinal haemorrhages are probably due to the diseases of the veins.

Where in the human eye does all the excess of the inflowing blood go, when one collapses the veins, or even the arteries during their diastole? It is accommodated in the capillary bed.

From this lengthy discussion it is evident that the basic structure of the vessels is the same in the eye as in other parts of the body, but modified to a certain degree in order to maintain the venous pressure constantly and more or less independently. We can verify all this theorisation by a simple experiment. Apply the cuff of the sphygmomanometer around the arm of a patient and raise the pressure to say $115 \mathrm{~mm}$. systolic. Soon the arm will become cyanotic, the veins gradually swell and the patient complain of increasing discomfort. Puncture the brachial vein with a large bore needle and leave it inside the vein. The blood will first gush out in a strong stream and then will be seen strong pulsations equal in number to the radial artery beating, but slightly later in order. The pulsation becomes less evident and finally the blood trickles out in frequent drops. The 
less pressure applied the less prominent are all these phenomena. It is the back pressure of the capillaries and arterioles that is brought into play by a venous obstruction.

If the reader finds all these details irksome, he should read through a discussion published a few years ago in the Brit. Med. $J l .^{10}$ where a paper by a distinguished cardiologist appeared, claiming that the brachial artery always remains open and never collapses during the measurement of the systolic blood pressure, because the colloids of the blood are non-compressible.

In intracranial pressure a similar problem is before us as in the case of the intra-ocular tension. We can only tell that the intracranial pressure is not higher than the intravenous pressure on the disc. In the very interesting book of Villaret, ${ }^{11}$ an idea is expressed that there may be two different venous pressures in the intracranial and in the peripheral venous systems. Records of direct measurements on animals are rare in this literature, but those of Binton, Op., are worth mentioning.

Is there any numerical connection between the general venous pressure as measured in the brachial vein and the retinal venous pressure? If, clinically at least, we can measure the arterial brachial pressure correctly, no such method was available until recently for the examination of the venous. Usually a small camera with a glass top was provided, the bottom being left exposed. It was placed on a prominent vein on either the hand, wrist or forearm, made as air-tight as possible, and then air was pumped in under a measured pressure and the veins carefully watched until the vein collapsed. The pressure of air at this point would indicate the intravenous pressure. It is obvious that this method allowed many mistakes to be made and, therefore, the figures of many observers varied greatly. Villaret recently produced a special apparatus whereby the intravenous pressure may be measured by a canula introduced into the cubital or ulnar vein of the patient and therefore carried out automatically under the conditions of a physiological experiment. Under these ideal conditions he came to the conclusion that the maximum value in normal cases is from 10 (female) to 14 (male) $\mathrm{cm}$. of water. As the normal intra-ocular tension is between $18-25 \mathrm{~mm}$. of mercury and the intravenous retinal pressure is almost equal we can see that the retinal is nearly 15-18 times higher than the brachial. This illustrates one of the most striking characteristics of the venous pressure as compared with the arterial. It is impossible to imagine the arterial pressure owing to the tremendous discrepancies normally present in different parts of the body. Has it ever been seen that a patient with $130 / 90$ in the brachial artery would normally show $260 / 180$ in the brain or any other part of 
the body? We will refer later on to its pathological importance. For the present I will only point out the fact that in many of my own cases I have measured the venous brachial pressure by Villaret's method in the same time as the retinal one was also measured. If no pathological factor was involved there was no correspondence whatsoever between the two, i.e., the venous pressure may be at its upper limit $14 \mathrm{~cm}$. of water, but the retinal intravenous tension was 15 and vice versa. For instance, in a man of 58 years of age with a blood pressure of $150 / 85$ with advanced sclerosis of the retinal vessels, the diastolic retinal pressure was 40 , the intra-ocular tension 25 and the brachial venous pressure $10 \mathrm{~cm}$. water. In a healthy young man of 34 with a blood pressure $120 / 90$, diastolic retinal pressure 25 , intra-ocular tension 20 , the brachial venous pressure was $8 \mathrm{~cm}$. of water. The autonomy of the eye and of its circulatory system was therefore maintained. It proved, however, to be different in the case of one patient who died from heart-failure. The rise of the brachial venous pressure may be caused by the influence of the central nervous system, or, more often, by a failing heart. There may also be other causes (altogether this much neglected branch of clinical medicine is still in its infancy). There is, however, nothing finer in our clinical armamentarium than the rise of venous brachial pressure as the earliest sign of cardiac failure. It subsides with the improvement of the heart, but if the rise be persistent it is a certain indication of a close dissolution. My patient was a man of 70 years of age with insufficient aortic and mitral valves, blood pressure $170 / 90$, retinal diastolic pressure 40 (by Bailliart's method), i.e., normal for him. The intra-ocular tension was $18 \mathrm{~mm}$. Hg. (Schiötz) intravenous brachial pressure $18 \mathrm{~cm}$. of water by Villaret's method. There were no clinical signs of heart failure but he complained of mild attacks of dyspnoea at night. Eight months later, at his own home, after a few days in bed his blood pressure was $150 / 20$, his liver enlarged and palpable, and his dyspnoea, even in the day-time, was distressing. The lungs were clear, the legs not oedematous. The brachial venous pressure was $20 \mathrm{~cm}$. of water-when he improved for a short time-it fell to $10 \mathrm{~cm}$., rising again to $18 \mathrm{~cm}$. (last examination three weeks before death from heart-failure). During the whole time the intra-ocular tension was $18-20 \mathrm{~mm}$. of mercury.

The possible nature of the spontaneous venous pulse.-Any explanation offered here would have to clarify the fact that (1) only one or two veins may be involved and the rest not at all ; (2) digital pressure or massage may transform the ordinary venous pulse into a spontaneous one in some veins, but not in others. All previous detailed investigation of the venous circulation of the eye leads to the conclusion that the cause of the spontaneous 
venous pulse must be a local one and not generally affecting all the veins. To this must be added the fact that on the retina, away from the disc, the vein will show the usual widening and narrowing of the trunk, but on the disc only the piston-like movement. The nearer the centre the clearer the piston-like movement of the spontaneous venous pulse will appear. This applies also to veins where external interference will produce the spontaneous pulse. If the vein bends sharply over the substance of the disc, or better still, over the artery just before it disappears from view, it may collapse and blanch, after expelling the blood. The spontaneous pulse is most pronounced if the vein dips into the disc perpendicularly or bends away from the observer at a more acute angle, i.e., an obtuse one towards the observer. As this is an invariable condition it is the probable cause. The spontaneous venous pulse will then be explained as an optical phenomenon just at the point where the vein is sharply bent. 'The curves of the arterial bends if sufficiently pronounced invariably pulsate in all patients. The veins even when they form similar waves (and this is practically limited to congenital tortuosity) only show the usual venous pulse. Then, however, their intravascular tension is very low indeed, as we have to deduct the intra-ocular tension, i.e., it may only be $1 \mathrm{~mm}$. of mercury if the bends are not sharp. In spontaneous vascular pulsation one has to deal with a real bend. Moreover the disc part of the vein is fixed and cannot be widened. The blood therefore has to pass through a sharp bend to squeeze itself from a wide into a narrower trunk and one watches the exact point of this bend. This means that the back pressure becomes more intense and the force of expulsion becomes greater. Hence the transformation of a peaceful rivulet into a miniature cascade.

If this explanation be correct then some deductions are obvious. The veins with ordinary venous pressure will have to bend sharply, but more deeply in the disc. They will then be in the same position as the veins of the spontaneous venous pulse. It is so and if one follows them deep into the disc one can usually see them pulsating with spontaneous pulsation. If one can imagine watching the eye from the inside of the brain one would probably see the spontaneous venous pulse on all the veins, as an obtuse angle seen from the cornea would mean an acute one from the brain. Therefore, two conditions are necessary for the appearance of the spontaneous venous pulse-a sharp bend in the vein not less than $90^{\circ}$; if it is much more than this the vein will collapse before the venous pulse appears. If one were able to bend all the veins to a certain angle they would all show the spontaneous venous pulse. In discussing all these points the position was simplified by allowing a direct route for the sharply bent vein 
towards the ophthalmic vein. But in reality some modifying factors interfere. In the substance of the disc the vein may not join the ophthalmic vein, but one of the branches of the central vein at a point where the latter does not yet possess the spontaneous venous pulse, or it may form some additional bends before joining the ophthalmic vein, etc. All this would tend to lessen the intensity of the spontaneous venous pulse and then digital pressure will produce one. The same applies to the central and even the ophthalmic vein where the intensity of the pulsation is diminished to some degree by the increased tortuosity of its course, or by some anatomical peculiarity. For instance, the central vein may be more or less narrowed when piercing the lamina cribrosa. I saw quite a healthy young girl with both veins joined in one trunk, the latter becoming distinctly narrower before going through the lamina. Fry examined 30 eyes microscopically. In only four cases he found a slight narrowing of the calibre of the veins when passing through the lamina. But at the point of exit from the nerve the vein may form acute angles (4-3) and its lumen may be compressed. The artery may also compress the vein when running together in the optic nerve. In my opinion the retinal vessels are under increased pressure on the papilla, covered by a special glial membrane. To all these modifying factors must be added the interference of the respiratory movements of the chest.

I allow myself some freedom in imagining the venous circulation of the brain. The venous blood leaves the eyeball under a pressure of at least $15-20 \mathrm{~mm}$. $\mathrm{Hg}$ on a wave-like track. It leaves the brain through the jugular veins where the pressure-in a sitting position-is still a few c.c. of water and in a recumbent position even less and possibly negative during the diastole of the heart and the inspiration of the chest. Moreover, the beats of the jugular venous pulse are exactly double those of the radial pulse corresponding to the diastole and systole of the heart, but sometimes varied by the respiratory movements of the chest. Most probably in the ophthalmic vein we have the same wave-like pulse. If this is so then all the venous circulation of the brain is carried on in a wave-like manner.

\section{Conclusion}

1. The spontaneous retinal venous pulse is seen only on the disc and is the intensification of the ordinary venous pulse depending upon the angle of the bend of the vein when entering the disc.

2. It is of no clinical significance whatsoever.

3. The venous retinal pulse vacillates round the intra-ocular tension. 
It is my pleasant duty to thank Mr. A. H. Levy for his usual benevolent criticism and help with the manuscript.

\title{
REFERENCES
}

1. Pines.-Sclerosis of the Retinal Vessels. Brit. Jl. of Ophthal., March, 1929.

2. BAILliart. - La circulat. ret. 1923.

3 Elliot.-The ret. pulse. Brit. Jl. of Ophthal., 1921.

4. Jauss.-Ueber den gefer. Arch.f. Ophthal., p. 437, 1929.

5. Kurzes Handb. der Ophthal., Vol. IV. J. Springer.

6. Eyster.-The clinical aspect of venous pressure. 1929. The MacMoll Cy.

7. BINTON.-Opift. Pflüger's Arch., Vol. CXXIX, p. 189, 1909.

8. SONDERMAN.-Arch. of Ophtal., p. 120, 1930.

9. Pines. Retinitis nephritica or albuminurica. Brit. Jl of Ophthal., 1931.

10. Harrington Sainsbury. - State of the arteries. Brit. Med. Jl., February, 1929.

11. Villaret.-La press. vein periph. Paris, 1930.

12. FRY.-Central vein of the retina. Arch. of Ophthal., 1930.

\section{LIGHTRESERVE FOR OCCUPATIONS IN SIGHT-SAVING CLASSES}

\author{
BY
}

\author{
Prof. R. A. KAZ, M.D.
}

(LENINGRAD)

IN young patients with defective sight much depends on physiotherapeutical treatment, and the steadiness of their improvement depends largely on the conditions of the sight-saving classes, and above all-on the sufficiency of light for occupations. The same, as I already said, is indicated by my measurer of lightreserve* through which the study or work are almost as visible as without the measurer. We, in the north, during the dark winter days find it is more difficult than in the south to receive with the measurer a positive result, particularly in feebly-seeing children with strong spectacles, which by themselves absorb a certain amount of light. So it is easy to conceive the various symptoms which can here arise-the symptoms of overfatigue of the eyes.

If, in spite of the last degree of fatigue which appears already in the breakdown of the eyes, in their burning, in temporal covering the object of reading, in endless winking of the eyelids, the worker

* The idea of reservelight as conservator of the working eyes was first put forward by me 40 years ago ("Reservelight as measurer of the sufficiency of the light for occupations." Vracz, 1897, No. 27), and for the last time-26 years ago ("Die Lichtvorratsprüfung in der Schulphotometrie." Internat. Arch. f. Schulhygiene, 1911). Of the works undertaken with my measurer of the lightreserve the investigations of $G$. J. Schoute merit particular attention which as a result of the School Commission determinated the sufficiency of light in the public-schools of Amsterdam (Geneeskundige Bladen, 1902). 